\title{
ANÁLISE DE UM MODELO INTELIGENTE DE CONTRATAÇÃO DE ENERGIA ELÉTRICA NO CURTO PRAZO PARA DISTRIBUIDORAS
}

\author{
Juan G. Lazo Lazo* \\ juandele.puc-rio.br
}

\author{
Karla Figueiredo* \\ karla@ele.puc-rio.br \\ *ICA/PUC-RJ \\ Rua Marquês de São \\ Vicente 225 \\ Gávea - RJ \\ PUC-RJ
}

\author{
Delberis Araujo Lima* \\ delberiscele.puc-rio.br
}

\section{ABSTRACT \\ Analysis of an Inteligent Model to Electricity Trading in the Short Term for Distribution Company \\ In this paper is presented a new approach of an intelligent model for optimization under uncertainty to determine the best strategy of electricity trading in the short term (referring to A-1 and Adjustment auctions) for distribution companies.}

This model reproduces all the rules of purchase/sale of energy for a distribution company and the transfer of this cost to the final tariff of the consumers. The optimization process uses genetic algorithm, and seeks to minimize the cost associated with the purchase of energy, penalty for subcontracting and the cost of trade (purchase/sale) energy by the spot price.

The optimal trading is obtained considering several load scenarios, obtained by Monte Carlo simulation, for a period of five years of analysis. The decisions of trading are taken in the first two years in that period.

The evaluation of the model results is done by means of a combination between the expected value of the distribution

Artigo submetido em 10/07/2011 (Id.: 1353)

Revisado em 14/09/2011, 12/12/2011, 29/02/2012

Aceito sob recomendação do Editor Associado Prof. Antonio Carlos Zambroni de Souza of costs and the $C V a R$ (Conditional Value at Risk), for the different load scenarios. The model also uses the PLD_robust, which seeks to minimize the exposure of the distribution company in the spot price.

To illustrate the results of the proposed model, a study case based on realistic data is presented. The results obtained are compared to the results obtained with the trading of energy without using the optimization model presented in this paper. That comparison is done to verify how much the proposed method can be better than the solutions based on intuitive analysis. In addition, further analysis is performed by considering two mechanisms of compensation of the surpluses and deficits of contracts, named MCSD4\% and MCSD_Expost, established by ANEEL to reduce the risks associated to the energy trading to the distribution companies.

KEYWORDS: Electricity trading, rules of the electrical system to the distribution companies, Optimization Model, Tariff of Energy.

\section{RESUMO}

Neste artigo é apresentada uma nova abordagem de um modelo inteligente de otimização sob incerteza para determinar a contratação de energia elétrica no curto prazo (referente aos leilões A-1 e Ajuste) para distribuidoras de energia elétrica. 
Nesse modelo estão incluídas todas as regras de contratação e repasse à tarifa, definidas pela ANEEL, para as distribuidoras. O processo de otimização utiliza um algoritmo genético, e busca minimizar o custo associado à contratação de energia elétrica, as penalidades por subcontratação e o custo da liquidação (compra/venda) desta energia ao PLD (Preço de Liquidação das Diferenças).

A contratação ótima é calculada considerando vários cenários de consumo, obtidos a partir de simulação Monte Carlo, para um período de cinco anos de análise. As decisões de contratação do modelo são tomadas nos dois primeiros anos desse período.

A avaliação dos resultados do sistema é feita considerando uma combinação entre o Valor Esperado (VE) da distribuição de custos e o CVaR (Conditional Value at Risk), para os diferentes cenários de consumo. O modelo também usa o PLD_robusto, que busca minimizar a exposição da distribuidora ao $P L D$.

Para ilustrar os resultados do modelo proposto é apresentado um estudo de caso baseado em dados reais. Os resultados obtidos são comparados com alguns resultados de contratação que não consideram o modelo de otimização proposto. Essa comparação é feita para se verificar o quanto o método proposto pode ser melhor que soluções baseadas apenas em análises intuitivas. Além disso, estudos adicionais são apresentados considerando os mecanismos de compensação de sobras e déficits, notadamente MCSD4\% e MCSD_Ex-post, previstos na legislação vigente do setor elétrico para minimizar os riscos associados à contratação de energia para as distribuidoras.

PALAVRAS-CHAVE: Contratação de energia elétrica, Regras de repasse de custo à tarifa do consumidor, Modelo de otimização e Tarifação de energia elétrica.

\section{INTRODUÇÃO}

Em 2004 o governo estabeleceu modificações importantes no modelo institucional do setor elétrico buscando aumentar a segurança no suprimento de energia, a modicidade tarifária e a universalização do atendimento aos consumidores. As bases do novo modelo consistem no planejamento de longo prazo, na coexistência dos ambientes de contratação regulada (ACR) e livre (ACL) e na premissa de que as distribuidoras devem prever e contratar energia suficiente para atender $100 \%$ do seu mercado. Com essas medidas, buscou-se incentivar investimentos em geração, de forma a garantir uma remuneração mínima para viabilizar tais investimentos (MME, 2003).
Nesse contexto, as distribuidoras devem contratar energia no ACR, por meio de leilões com prazos definidos de entrega dessa energia (CCEE, 2010). Os dispositivos legais usados para orientar essa contratação incentivam fortemente as distribuidoras na contratação de, pelo menos, $100 \%$ de sua carga passível de repasse ${ }^{1}$ à tarifa. Isso ocorre porque a contratação de uma quantidade de energia inferior à carga regulatória expõe a distribuidora a contratar energia ao valor do $P L D$ (Preço de liquidação de diferenças) e ao pagamento de elevadas multas por subcontratação. Por outro lado, a contratação de uma quantidade de energia superior à carga regulatória, expõe a distribuidora a vender o excesso de energia ao valor do $P L D$, já que o repasse à tarifa devido à sobre-contratação está limitado, por dispositivos legais, a 103\% da carga regulatória.

Com as novas medidas estabelecidas no setor elétrico, surgiram desafios importantes para as distribuidoras. O principal deles é a previsão de carga. As distribuidoras devem prever sua carga para um período longo de tempo (vários anos) e, com isso, definir a melhor estratégia de contratação da energia para suprimento dessa carga.

Outro desafio importante é, estimado o consumo (carga), determinar qual a contratação ótima da distribuidora que minimiza o custo associado às penalidades e prejuízos por subcontratação e sobre-contratação.

Várias têm sido as propostas apresentadas para otimizar a contratação da distribuidora, no curto e longo prazo. Em (CASTRO, 2004a) é apresentada uma análise para avaliar os riscos associados às distribuidoras no novo modelo do setor elétrico.

Em (CASTRO, 2004b) são analisados os riscos de uma distribuidora estar exposta na contratação de energia frente às incertezas (consumo de energia elétrica e variação de preços nos leilões) do mercado.

Outro trabalho, desenvolvido por (AZEVEDO, 2004) utiliza a teoria de jogos não cooperativos para avaliar as estratégias de contratação de energia elétrica de geradores e distribuidoras no novo ambiente de comercialização de energia elétrica.

Em (GUIMARÃES, 2005) é apresentado um modelo estocástico para contratação de energia das distribuidoras. A metodologia de solução utiliza otimização estocástica multiestágio, e leva em consideração os diversos horizontes de contratação e preços da energia, visando minimizar uma ponderação entre tarifa para consumidor e custos para distribuidora.

\footnotetext{
${ }^{1}$ A carga passível de repasse, também conhecida como carga regulatória, inclui o mercado faturado da distribuidora mais as perdas elétricas regulatórias, que são a soma das perdas elétricas técnicas e não-técnicas da distribuidora, passíveis de repasse.
} 
Em (SUSTERAS, 2006) é apresentada uma ferramenta de apoio à decisão para os geradores, simulando o comportamento das distribuidoras a partir de uma proposta baseada em algoritmo genético.

Já em (RODRIGUES, 2007) é apresentado um modelo computacional para planejamento de contratação de energia elétrica de concessionárias de distribuição do Brasil para um horizonte de curto/curtíssimo prazo de modo a se obter a menor despesa com a contratação de energia para atendimento de sua demanda, com ênfase na compra de energia de unidades de geração distribuída. Para tratamento de incertezas na geração e demanda, nesse caso, foi utilizada programação linear fuzzy.

No trabalho de (OLIVEIRA, 2009) é feita uma proposta de otimização de portfólios de contratos de energia de uma distribuidora considerando a fronteira eficiente. A técnica apresentada utiliza o valor esperado e o $C V a R$ como medida de risco.

Trabalhos recentes que visam otimizar contratos de energia elétrica utilizando o $C V a R$, também podem ser encontrados em (TORRES, 2006), (BENATI, 2007), (HUANG, 2008), (GUDER, 2009), (STREET, 2009) e (BARROSO, 2011).

Finalmente, em (LAZO, 2010) foi apresentado uma versão parcial do modelo proposto neste trabalho. Neste artigo, propõe-se analisar o modelo de otimização para definir a melhor estratégia de contratação de energia elétrica, considerando um horizonte de 2 (dois) anos para as decisões de contratação e 5 (cinco) anos para analisar o efeito dessas decisões. A estimação da carga por parte da distribuidora não faz parte do escopo deste artigo, sendo considerados vários cenários de carga, gerados através de simulação Monte Carlo, considerando três cenários realistas de consumo (médio, otimista e pessimista), fornecidos por uma distribuidora.

Para avaliar a qualidade dos resultados associados à contratação de energia com esse modelo, propõe-se compará-lo com uma estratégia de contratação baseada em decisões intuitivas. Nessas decisões são analisados diferentes valores de possíveis contratações para os leilões de Ajuste e A-1, e são calculados os custos da distribuidora para esses valores. Em seguida, os melhores resultados são comparados com o resultado do modelo de otimização proposto. A comparação dos resultados é feita levando-se em conta todas as regras de contratação e repasse às tarifas determinadas pela ANEEL (DECRETO, 2004). Para comparar os resultados das diferentes estratégias de contratação, levando-se em conta os diferentes cenários de carga, será utilizado uma métrica baseada na ponderação entre o Valor Esperado e o CVaR (Conditional Value at Risk), que é uma medida usada para simular diferentes níveis de aversão à risco.
Além disso, é avaliado o impacto dos mecanismos de mitigação de risco de contratação que dispõem as distribuidoras: MCSD4\% (Mecanismo de contratação de sobras e déficit limitado a 4\%) e MCSD Ex-post (Mecanismo de contratação de sobras e déficit para ajuste financeiro). A apresentação dos dados de entrada e resultados é feita usando a interface gráfica do modelo apresentado.

Podem-se resumir as principais contribuições do trabalho proposto da seguinte forma:

1. A proposta de um modelo inteligente de contratação de energia elétrica em leilões de curto prazo: $\mathrm{O}$ modelo proposto utiliza vários cenários de carga e, como medida de avaliação, uma combinação entre o Valor Esperado da distribuição e o $C V a R$. Isso permite que usuários com diferentes níveis de aversão ao risco possam usar a ferramenta proposta;

2. O uso do PLD_robusto: O PLD (preço de liquidação das diferenças) é um dos resultados do problema de otimização, obtido a partir do despacho ótimo de energia estabelecido pelo ONS (Operador Nacional do Sistema). O PLD determina o custo de energia para distribuidoras que não contrataram energia suficiente para suprir sua carga. Para buscar minimizar a exposição da distribuidora ao $P L D$, neste trabalho usa-se o PLD_robusto, que estabelece valores máximos e mínimos de $P L D$ e que induzem uma decisão na qual a distribuidora sempre buscará estar contratada, evitando a exposição ao $P L D$. Dessa forma, o modelo proposto neste trabalho pode ser desacoplado dos cenários de $P L D$ gerados pelo ONS.

3. Um estudo quantitativo para verificar a importância dos mecanismos de compensação de sobras e déficits (MCSD) para as distribuidoras e o efeito sobre as decisões de contratação.

4. A apresentação da interface gráfica do modelo, que permite aos usuários inserir dados e analisar resultados de forma simples e didática.

Para alcançar os objetivos propostos, este artigo está dividido da seguinte forma: na seção 2 está apresentado o modelo do sistema proposto para contratação de energia elétrica; na seção 3 é apresentado um estudo de caso para comparar os resultados da contratação usando o sistema proposto com outras decisões intuitivas; na seção 4 está apresentada a conclusão do trabalho.

\section{MODELO DE OTIMIZAÇÃO}

Nesta seção é apresentado, em detalhes, o modelo proposto para determinar a contratação ótima da distribuidora nos lei- 
lões de curto prazo. O modelo proposto está baseado em otimização estocástica, que tem sido largamente utilizado para solução de contratação de energia no setor elétrico, e utiliza o CVaR como medida de risco para avaliar os diferentes cenários simulados.

Na figura 1 está ilustrado o diagrama de blocos do modelo. Cada bloco apresentado no diagrama está referenciado por um número. Esses blocos são detalhados nas subseções seguintes.

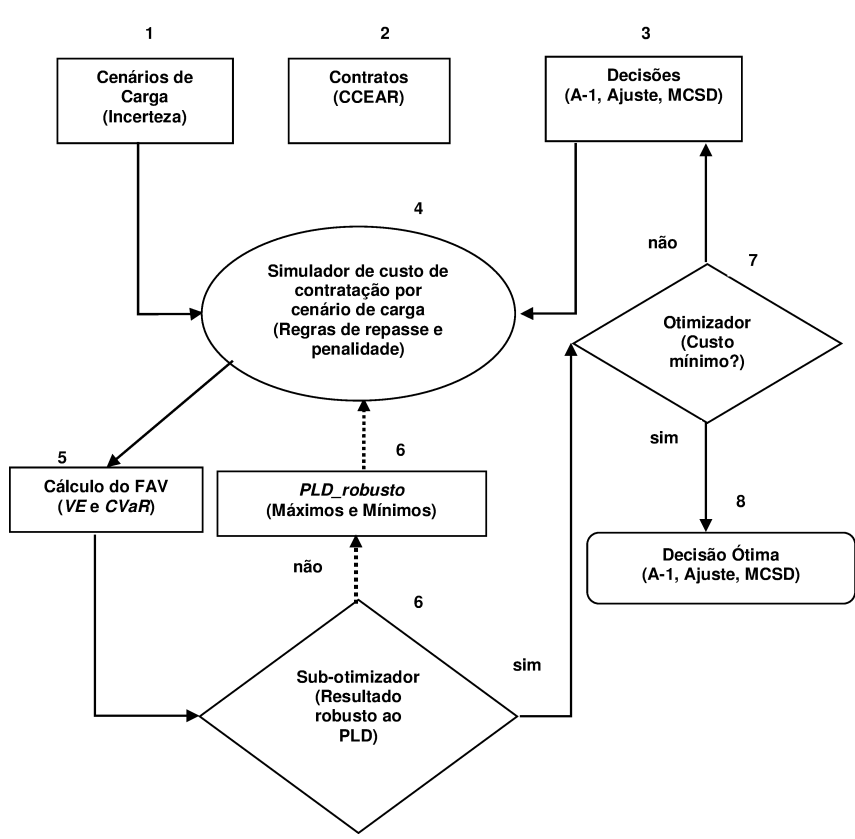

Figura 1: Diagrama de blocos do sistema proposto.

\subsection{Cenários de carga (Bloco 1)}

Neste trabalho, utilizamos a Simulação Monte Carlo para geração de cenários de carga. A utilização da Simulação Monte Carlo para geração de cenários de carga é adequada, pois se trata de uma técnica orientada para solução de problemas que possuem uma natureza probabilística (estocástica). Algumas aplicações da Simulação Monte Carlo podem ser vistas nos mercados financeiros. O tema foi analisado em (SILVA, 1998) e (JORION, 1998). Com a introdução dos mercados de energia elétrica, os estudos envolvendo simulações estocásticas incorporaram essa técnica para simulação de cenários futuros de carga, preços, entre outras variáveis que tem uma natureza estocástica. Em (ISHIHARA, 2002) é apresentado um exemplo da incorporação da técnica para mensurar os riscos na comercialização de energia elétrica. Trabalhos que combinam a simulação Monte Carlo com aplicação di- reta do $C V a R$ para otimização de carteira de contratos, como usados neste trabalho, podem ser encontrados também em (ARTZNER, 1999), (LU, 2007) e (HUANG, 2008).

No modelo proposto neste artigo são gerados vários cenários de carga da distribuidora para os cinco anos seguintes ao ano inicial de análise. Os cenários são gerados a partir de projeções média, pessimista e otimista, pré-estabelecidas a partir de dados reais fornecidos por uma distribuidora.

Para a geração dos cenários foi utilizada a técnica Quase Monte Carlo de Sobol (SOBOL, 1967), (BOYLE,1997), (BIRGE,1995) e (WANG,2001), que permite uma eficiente distribuição das amostras aleatória sobre o espaço de busca. Assim, consegue-se reduzir significativamente o número de amostras necessárias para realizar a simulação Monte Carlo.

Além disso, para o estudo proposto no artigo, foi considerado uma distribuição triangular de probabilidade para geração de cenários, em que os valores dessa distribuição são obtidos em cada mês, considerando-se as projeções média, pessimista e otimista fornecidas pela distribuidora. Dessa forma, cada mês terá uma distribuição triangular com parâmetros diferentes. Para ilustrar, na figura 2 está apresentada como se dá a geração de cenários (mês a mês) para os cinco primeiros meses de análises da distribuidora estudada.

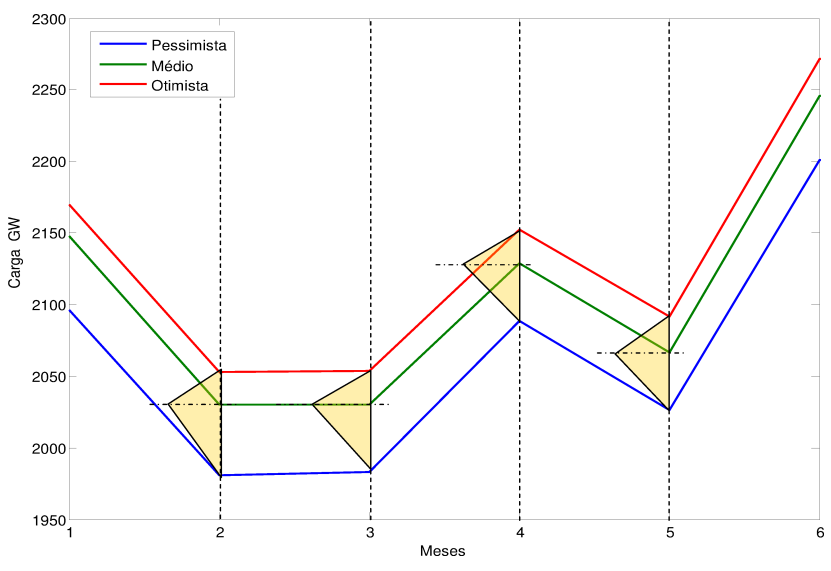

Figura 2: Projeções de carga e construção das distribuições para os cenários de carga.

\subsection{Contratos (Bloco 2)}

Para estabelecer regras na contratação de energia através dos CCEAR (Contratos de Comercialização de Energia no Ambiente Regulado), os contratos das distribuidoras são separados por produtos. Esses produtos são determinados por tipo de geração no qual são estabelecidos os contratos (CCEE, 2010). 
Neste trabalho, os contratos existentes são contratos vigentes no período de análise considerado e são tomados como dados de entrada. Os dados de entrada de cada contrato são: quantidade de energia adquirida $(\mathrm{GWh})$, preço da energia (R\$/MWh), leilão em que foi adquirida a energia, a procedência da energia (nova, existente, leilão de Ajuste, MCSD, etc.), a data do leilão, a data de início do fornecimento da energia, a data do término do contrato e o índice de reajuste de preço da energia a ser consumida. Neste artigo, esses contratos existentes formam a base dos contratos sobre os quais as novas decisões de contratação são tomadas.

\subsection{Decisões (Bloco 3)}

As decisões são o resultado final do processo de otimização produzido pelo modelo. As variáveis de decisão do modelo proposto são: quantidade de energia $(\mathrm{GWh})$ a ser contratada nos leilões A-1 e Ajuste, o percentual de contratação ou descontratação no MCSD4\%. As variáveis de decisão serão tomadas em função de uma medida de custo (em R\$) associada aos vários cenários de carga projetados.

Neste artigo, as decisões de contratação relacionadas aos leilões de energia de longo prazo (A-3 e A-5), ainda que sejam incertas, são consideradas conhecidas. Além disso, caso esses contratos terminem no período de análise, considera-se que a mesma quantidade de energia é recontratada, de acordo com a legislação vigente.

Outro elemento importante nas variáveis de entrada é a estimação de um intervalo de segurança de contratação para os leilões de Ajuste e A-1. Esse dado de entrada deve estabelecer um intervalo de oferta de energia e o respectivo preço estimado para essa energia de forma que o sistema possa identificar quais os melhores valores de contratação.

\subsection{Simulador de custo (Bloco 4)}

O bloco 4 simula o custo de contratação associado à distribuidora, segundo as regras vigentes do setor elétrico (CCEE, 2010).

Por simplicidade, nesta seção são apresentadas apenas as regras que foram ativadas nesse estudo para determinar o custo, já com a simulação do repasse à tarifa do consumidor por parte da distribuidora. O custo da distribuidora, obtido para cada cenário de carga $c$ e associado a uma decisão de contratação $i$, considerando um período de análise de $m$ meses pode ser obtido por:

$$
\begin{aligned}
C_{m}^{i, c} & =C_{\text {cont. }-m}^{i}+C_{\text {subcont }-m}^{i, c}+C_{\text {pen } .-a}^{i, c}- \\
& -R_{\text {cont. }-m}^{i, c}-R_{\text {sobrecont. }-m}^{i, c}
\end{aligned}
$$

O primeiro termo da expressão (1) representa o custo associado a uma decisão de contratação $i$ qualquer, para um período de análise de $m$ meses, e pode ser obtido por:

$$
C_{\text {cont. }-m}^{i}=\sum_{t=1}^{m}\left(P_{t} \cdot E_{t}^{i}\right) \cdot\left(1+K_{m}\right)^{-t}
$$

Sendo:

$P_{t}$ - Preço do contrato (em R $\$ / M W h$ ) de energia no mês $t$, ajustado pelo índice correspondente ${ }^{2}$.

$E_{t}^{i}$ - Fração da energia total $\left(E^{i}+E^{e x}\right)$ sazonalizada no mês $t$, devido à decisão de contratação $i$, em MWh;

$E^{i}$ - Energia total contratada e/ou descontratada (em MWh), devido a decisão $i$;

$E^{e x}$ - Energia total (em MWh) dos contratos existentes;

$K_{m}$ - Taxa de juros mensal, para o período de $m$ meses.

A variável de decisão $E^{i}$ representa o resultado da energia contratada (ou descontratada) ao longo do período de decisão. Essa variável representa a soma da energia contratada nos leilões A-1 (para primeiro e segundo ano), a energia contratada nos leilões de Ajuste (para primeiro e segundo ano) e a energia contratada (ou descontratada) no MCSD4\%. Essa energia contratada mais a energia existente são sazonalizadas $\left(E_{t}^{i}\right)$ para cada período $t$. A decisão de sazonalização não faz parte do escopo deste artigo, sendo tomada como um dado de entrada.

O segundo termo de (1) representa o custo de subcontratação associado a uma decisão de contratação qualquer $i$, ao cenário de carga $c$, para um período de análise de $m$ meses, conforme mostra a equação (3):

$$
\begin{gathered}
C_{\text {subcont. }-m}^{i, c}=\sum_{t=1}^{m}\left(L_{t}^{c}-E_{t}^{i}\right) \cdot \pi_{r}^{\max } \cdot\left(1+K_{m}\right)^{-t}, \\
L_{t}^{c}>E_{t}^{i}
\end{gathered}
$$

Sendo:

$L_{t}^{C}$ - Carga (em MWh) passível de repasse no mês $t$;

$\pi_{r}^{\max }$ - Preço de liquidação de diferenças robusto (PLD_robusto) máximo, dado em R $\$ / \mathrm{MWh}$.

O terceiro termo da expressão (1) representa o custo da penalidade por subcontratação associado a uma decisão de con-

\footnotetext{
${ }^{2}$ Cada contrato tem seu índice de reajuste de preço. Para o sistema proposto, considera-se que o índice de reajuste de preço dos contratos é o IPCA (Índice de Preços ao Consumidor Amplo).
} 
tratação qualquer $i$, ao cenário de carga $c$, para um período de análise de $a$ anos, conforme mostra a equação (4):

$$
\begin{aligned}
& C_{\text {pen. }-a}^{i, c}=\sum_{t=1}^{a}\left(\text { Lano }_{t}^{c}-\text { Eano }_{t}^{i}\right) . \\
& {\left[\operatorname{Max}\left(P L D_{t} ; V R_{t}\right)\right] \cdot\left(1+K_{a}\right)^{-t} \text {, }} \\
& \text { Lano }_{t}^{c}>\text { Eano }_{t}^{i}
\end{aligned}
$$

Sendo:

$\operatorname{Lano}_{t}^{c}$ - Carga (MWh) no ano $t$;

$E^{E a n o}{ }_{t}^{i}$ - Energia contratada (MWh) associada a decisão $i$, para o ano $t ; \operatorname{Max}\left(P L D_{t}\right)$ - Maior valor de $P L D(\mathrm{R} \$ / \mathrm{MWh})$ no ano $t$;

$\operatorname{Max}\left(V R_{t}\right)$ - Maior valor anual de referência ${ }^{3}(\mathrm{R} \$ / \mathrm{MWh})$ no ano $t$.

$K_{a}$ - Taxa de juros anual, para o período de $a$ anos.

Na expressão (4) o custo de penalidade é calculado com base anual porque a penalidade por subcontratação é computada apenas no final de cada ano.

O quarto termo da expressão (1) representa a receita da distribuidora associada a uma decisão de contratação $i$, para um período de análise de $m$ meses, conforme mostra a equação (5):

$$
\begin{gathered}
R_{\text {cont. }-m}^{i, c}=\sum_{t=1}^{m}\left(\text { PLDrep }_{t} \cdot E_{t}^{i}\right) \cdot\left(1+K_{m}\right)^{-t}, \\
E_{t}^{i} \leq 103 \% \cdot L_{t}^{c}
\end{gathered}
$$

Sendo:

PLDrep - Preço de repasse ${ }^{4}(\mathrm{R} \$ / \mathrm{MWh})$ associado ao período $t$.

$\mathrm{Na}$ expressão (5) está estabelecido que o repasse a tarifa devido à contratação de energia está condicionado a $103 \%$ da carga no mês de análise, conforme estabelecido pelas regras vigentes no setor elétrico (DECRETO, 2004).

O quinto termo da expressão (1) representa a receita da distribuidora associada a uma decisão de contratação $(i)$, para um período de análise de $m$ meses devido a sobrecontratação

\footnotetext{
${ }^{3} \mathrm{O}$ Valor de referência $(V R)$ corresponde à média, calculada com base mensal, apurada dos preços de energia elétrica nos leilões "A-5" e "A-3", calculados para todas as empresas de distribuição.

${ }^{4} \mathrm{O}$ preço de repasse da distribuidora é usado para simular o efeito de repassar a energia contratada à tarifa do consumidor final. A obtenção desse preço não faz parte do escopo do trabalho, sendo usado o valor fornecido pela distribuidora para o período de estudo.
}

acima de $103 \%$ da carga da distribuidora, conforme mostra a equação (6):

$$
\begin{aligned}
& R_{\text {sobrecont }-\mathrm{m}}^{i, c}=\sum_{t=1}^{m}\left(E_{t}^{i}-103 \% L_{t}^{i}\right) \cdot \pi_{r}^{m i n} \cdot\left(1+K_{m}\right)^{-t} ; \\
& E_{t}^{i}>103 \% L_{t}^{i}
\end{aligned}
$$

Sendo:

$\pi_{r}^{\text {min }}$ - Preço de liquidação de diferenças $(P L D)$ robusto mínimo, dado em R $\$$ /MWh.

\subsection{Função de avaliação (FAV) ( Bloco 5)}

Neste trabalho, a medida que estabelece a qualidade das decisões produzidas pelo sistema é determinada pelo custo da distribuidora obtido para os vários cenários de carga $c$ projetados para todo o período de análise, e representa a função de avaliação do modelo.

Para levar em conta todos os cenários de carga, essa função considera o Valor Esperado dos custos produzidos pelo modelo de todos os resultados associados a cada cenário de carga determinados na seção anterior e o $C V a R$, calculado como a média dos $x \%$ piores cenários. Ambos os resultados (VE e $C V a R$ ) são combinados para gerar uma única medida de custo, apresentada na expressão (7):

$$
\text { Custo }^{i}=\alpha \cdot C_{m}^{i}+(1-\alpha) \cdot C V a R_{x \%}^{i}
$$

Sendo:

Custo $^{i}$ - Medida de custo (em R \$) da distribuidora associado à decisão $i$;

$\alpha$ - Fator de aversão ao risco;

$C_{m}^{i}=\frac{\sum_{c=1}^{n} C_{m}^{i, c}}{n}$ - Custo médio associado à decisão $i$ e aos diversos cenários de carga;

$C V a R_{x \%}^{i}$ - Custo associado à decisão $i$, obtido pela média dos $x \%$ piores cenários;

$n$ - Número de cenários de carga considerados.

O $C V a R$ tem recebido maior atenção na última década tanto por acadêmicos, como pela indústria. Os principais motivos por esse interesse são: (i) a sua intuitiva interpretação econômica, que com uma ligeira redefinição pode ser entendido como uma medida de valor, como apresentado em (STREET, 2009); (ii) o seu enquadramento como uma medida coerente de risco, que se caracteriza por quatro propriedades desejáveis de uma medida de risco (ARTZNER, 1999) e, por fim; 
(iii) a sua forma funcional escrita como um problema de otimização convexo, o que proporciona uma grande vantagem algorítmica pois não compromete a convexidade de problemas convexos ao considerar tal medida como métrica de otimização (ROCKAFELLAR, 2000). Além disso, assim como em (ROCKAFELLAR, 2000), o CVaR, por se tratar de uma medida de risco, será usado neste trabalho como a média dos $x \%$ piores cenários de perdas financeiras.

\subsection{PLD Robusto (Bloco 6)}

Os valores de $P L D$ usados neste modelo para induzir a distribuidora manter-se contratada dentro da faixa de contratação passível de repasse (ver expressões (3) e (6)) são chamados PLD_Robusto. Para considerar o PLD_robusto, utilizam-se apenas dois valores de $P L D$ : um máximo $\left(\pi_{r}^{\max }\right)$ e um mí$\operatorname{nimo}\left(\pi_{r}^{\min }\right)$.

$\mathrm{O} \pi_{r}^{\max }$ é utilizado nas situações em que a distribuidora, com as decisões de contratação já tomadas, e a sazonalização previamente definida, necessita adquirir mais energia para atender sua carga. Assim, essa energia extra será adquirida ao valor de $\pi_{r}^{\max }$. O $\pi_{r}^{\text {min }}$ é utilizado nas situações em que a distribuidora, com as decisões de contratação já tomadas e a sazonalização previamente definida, necessita vender o excesso de energia ao PLD. Esse excesso de energia será vendido ao valor de $\pi_{r}^{\min }$

Então, haverá um estimulo natural para aquisição de contratos suficientes para atender a carga, evitando a sobre e subcontratação. Com isso, a solução apresentada é desacoplada dos cenários reais de $P L D s$, já que considera apenas valores máximo $\left(\pi_{r}^{\max }\right)$ e mínimo $\left(\pi_{r}^{\min }\right)$.

\subsection{Otimizador (Bloco 7)}

Todo o processo de otimização de contratos é desenvolvido a partir de um algoritmo genético (GOLDBERG, 1989), (MICHALEWICZ, 1996). O fim do processo de otimização é atingido quando a evolução dos resultados (medida de custo associado à contratação) passa a ser muito pequena a cada iteração, ou quando o algoritmo atingir um número máximo de gerações. O modelo de otimização utilizando o algoritmo genético proposto para solução do problema é descrito em detalhes nas subseções a seguir.

\subsubsection{Codificação do Problema}

Neste trabalho, as variáveis de decisão são a quantidade de energia a ser adquirida nos leilões de Ajuste e A-1, para o primeiro e segundo ano de decisão, bem como a quantidade de energia a ser contratada e/ou descontratada através do MCSD4\%. A figura 3 representa a forma de codificação das variáveis para o primeiro e segundo ano de decisão.

\begin{tabular}{|c|c|c|c|c|c|}
\hline A-1 & Ajuste & MCSD4\% & A-1 & Ajuste & MCSD4\% \\
\hline
\end{tabular}

Figura 3: Representação de um indivíduo do problema proposto.

A representação escolhida para codificar as variáveis é a representação por números reais. Essa representação é mais rápida, pois evita a decodificação. Também tem a propriedade de que dois pontos (indivíduos) próximos um ao outro no espaço de representação, estão também próximos no espaço de solução do problema.

\subsubsection{População}

O tamanho da população está relacionado com o desempenho global e a eficiência dos AGs. Para o problema apresentado utilizou-se uma população de 40 indivíduos, visto que acima dessa população os resultados não são significativamente alterados. Para iniciar o processo de análise, a população inicial é gerada de forma aleatória.

\subsubsection{Função de avaliação}

A função de avaliação representa a aptidão (ou qualidade) de um individuo, ou seja, indica o quanto boa é a solução do problema. Neste trabalho, seu valor é determinado por uma medida de custo da distribuidora associada aos vários cenários de carga considerados. Quanto menor o valor dessa medida, mais bem adaptado é o indivíduo. Assim, a função de avaliação será obtida pela expressão (7), que determina o resultado de uma decisão de contratação associada à composição de vários cenários de carga.

\subsubsection{Seleção}

Na seleção, membros da população são escolhidos para aplicação de operadores genéticos. O processo de seleção usado neste trabalho é chamado roleta. Esse processo consiste em selecionar os indivíduos por meio de uma "roleta" na qual os indivíduos mais bem adaptados (soluções com os custos baixos) têm maiores chances de serem selecionados para realizar as operações de cruzamento e mutação. 


\subsubsection{Elitismo}

Para que os melhores indivíduos não sejam eliminados de uma geração para outra, aplica-se o operador elitismo. Isso garante que os melhores indivíduos de uma geração sejam mantidos automaticamente para a próxima geração. Neste trabalho, depois de considerar diferentes possibilidades, optou-se por utilizar um valor de elitismo igual a dois, ou seja, os dois melhores resultados obtidos em uma geração são mantidos na próxima geração.

\subsubsection{Cruzamento}

O operador cruzamento (recombinação) possibilita a criação de novos indivíduos através da combinação de dois ou mais indivíduos e, com isso, obtém indivíduos mais bem adaptados. Neste trabalho, foi usado o cruzamento aritmético entre dois pontos, com taxa de $65 \%$.

\subsubsection{Mutação}

O operador mutação tem como objetivo manter a diversidade da população. Esse operador modifica de forma aleatória um ou mais genes do cromossomo, de acordo com uma probabilidade denominada taxa de mutação. A mutação adotada no trabalho foi a mutação gaussiana, com taxa de $1 \%$.

\subsubsection{Critério de Parada}

O critério de parada do algoritmo genético ocorre quando a variação do resultado da função objetivo de uma iteração para outra é muito pequena ou quando o algoritmo genético atinge 50 gerações.

\subsection{Decisão Ótima (Bloco 8)}

A decisão ótima são os valores obtidos para a contratação de energia (figura 3), para o qual a função de avaliação tem o menor custo possível.

\section{ESTUDO DE CASO}

Este estudo de caso consiste em avaliar os resultados da decisão de contratação de energia obtidos a partir do modelo de contratação apresentado na figura 1 . Os resultados apresentados nesta seção são obtidos considerando um portfólio de contratos e um perfil de consumo realista de uma distribuidora. Na figura 4 é ilustrado um ano típico da distribuidora estudada no qual os contratos (existentes) dessa distribuidora são sazonalizados ao longo de um ano, com base na energia total contratada para este ano. Também é apresentado o perfil da carga com relação à carga total deste mesmo ano de estudo.
Para avaliar o modelo proposto neste trabalho, os resultados apresentados neste estudo de caso são divididos em duas partes: na primeira parte considera-se que a distribuidora não dispõe do modelo proposto para otimização de contratos e decisões intuitivas são tomadas. Na segunda parte, considera-se o uso do modelo proposto para as decisões de contratação.

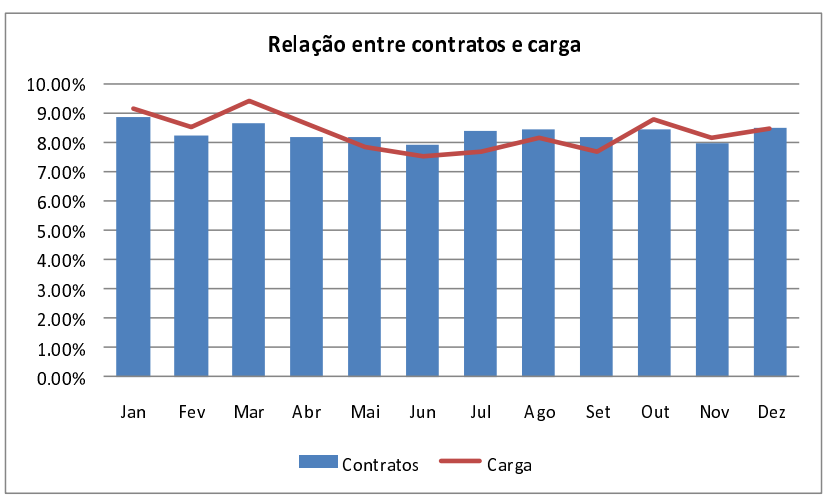

Figura 4: Relação entre contratos existentes e a carga da distribuidora ao longo de um ano.

Na primeira parte, as decisões de contratação nos leilões, A-1 e Ajuste, são fixadas (sem otimização) em diferentes patamares de contratação e é avaliada a medida de custo associado a cada uma dessas decisões. O objetivo desse teste é reproduzir o comportamento de um agente que não utiliza a ferramenta proposta para contratação de energia.

Na segunda parte, é utilizado o modelo de otimização para obtenção da quantidade ótima de contratos A-1 e Ajuste, que minimiza a medida de custo (equivalentemente, maximiza a medida de lucro) da distribuidora. Além disso, são utilizados os mecanismos de mitigação de riscos: MCSD4\% e o MCSD Ex-post, dos quais o MCSD4\% também é otimizado.

O MCSD4\% é um mecanismo que pode ser usado pelas distribuidoras para devolver parte da sua energia (até $4 \%$ da energia de alguns contratos) de forma unilateral. Nesse caso, a distribuidora, em função de eventuais quedas inesperadas no consumo de energia pode usar essa ferramenta. Também pode ser usado para aquisição de contratos (até 4\% da energia de alguns contratos existentes). Esse mecanismo é importante para as distribuidoras devido ao longo prazo de vigência dos contratos e da incerteza na carga futura. O MCSD_Expost é um mecanismo financeiro que permite que distribuidoras que estejam sobrecontratadas, ao final de um ano de apuração, repassem contratos a distribuidoras que estejam subcontratadas. 


\subsection{Premissas usadas para o estudo de caso}

Nos testes realizados, a quantidade de energia máxima a ser contratada está limitada por valores pré-estabelecidos. Para os leilões A-1, definiu-se que a contratação de energia, para cada ano de decisão, está condicionada a um valor máximo de $1000 \mathrm{GWh}$, para um período de análise de cinco anos. O preço da energia elétrica para compra nos leilões A-1 é de $80 \mathrm{R} \$ / \mathrm{MWh}$ para o primeiro ano e $100 \mathrm{R} \$ \mathrm{MWh}$ para o segundo ano. Na figura 5, usada na interface do modelo proposto, são apresentadas detalhadamente as informações que determinam a contratação dos leilões A-1, para o primeiro e segundo ano.

Para os leilões de Ajuste, a quantidade máxima de energia possível de ser contratada será de $400 \mathrm{GWh}$ para cada ano de decisão a um custo de 95 R $\$$ MWh, para o primeiro ano, e 110 R\$/MWh para o segundo ano. Nas figuras 6 e 7 estão apresentados os parâmetros da contratação referente à modalidade ajuste, para o primeiro e segundo ano, respectivamente.

No modelo baseado em otimização robusta, considera-se que, para cada mês do ano, caso haja sobra de energia por parte da distribuidora, essa energia seja vendida ao valor do $\pi_{r}^{\min } \mathrm{e}$, havendo falta de energia, a energia seja comprada ao valor de $\pi_{r}^{\max }$. No estudo proposto os valores de PLD_Robusto são: 400,00 R \$/MWh $\left(\pi_{r}^{\max }\right)$ para compra e 20,00 R\$/MWh $\left(\pi_{r}^{\text {min }}\right)$ para venda.

O MCSD4\%, para o nosso estudo, está limitado a 4\%. A aplicação do MCSD4\% para os dois primeiros anos está condicionada a um período de vigência estabelecido pelos contratos sobre os quais o MCSD atua. O preço da energia contratada no MCSD4\% é de 50,00 R\$/MWh e a descontratação terá valor de energia igual ao preço da energia contratada.

Para a simulação, são gerados 500 cenários de carga aleatoriamente, dentro de limites de cargas máxima e mínima de cada mês, previstos pela distribuidora estudada, para os cinco anos de análise. Para o problema em questão, verificou-se que os 500 cenários de carga são suficiente para obter uma boa amostragem e convergência da simulação, por isso foi utilizada a técnica Quase Monte Carlo de Sobol para a geração dos cenários, e porque acima deste valor verificou-se que não há mudanças significativas nos resultados. Entretanto, deve-se enfatizar que o modelo proposto admite que se trabalhe com um número qualquer de cenários.

Simulou-se que, dado o valor médio de carga fornecido pela distribuidora, a carga poderia variar em intervalos limitados pelos valores máximos e mínimos previstos pela distribuidora (ver figura 2). Na figura 8 estão detalhados os parâmetros usados para aplicação do MCSD4\%, bem como a inter- face usada no modelo para escolha do processo de otimização.

Todos os parâmetros apresentados foram considerados baseando-se em valores históricos de leilões e dados realistas da distribuidora estudada. A sazonalização dos contratos utilizados no trabalho ao longo dos anos foi feita com base na sazonalização dos contratos do ano de 2008 da distribuidora, com perfil apresentado seguindo o padrão figura 4 .

Para considerar os reajustes de preços dos contratos ao longo dos anos, utilizou-se o IPCA (Índice de Preços ao Consumidor Amplo, projetado para os cinco anos da análise). Para que não haja influência das decisões de longo prazo nas análises, considera-se que a energia proveniente de leilões de longo prazo (A-5 e A-3) será recontratada, caso esses contratos terminem dentro do período de análise considerado. $\mathrm{O}$ mesmo critério foi usado para os contratos de energia existente.

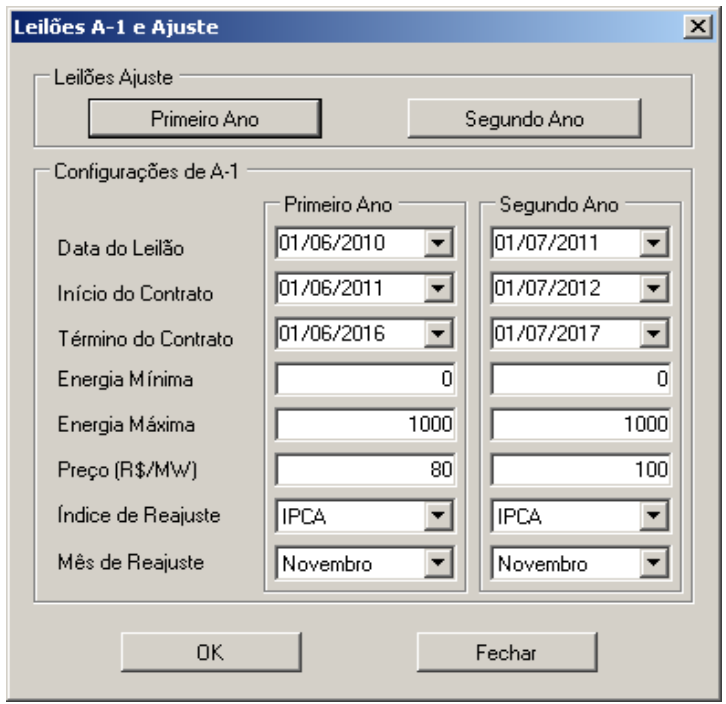

Figura 5: Interface gráfica com os parâmetros dos leilões A-1 no primeiro e segundo ano de decisão.

Para o estudo proposto, escolheu-se variável $\alpha$, da expressão (7), como 5\%, o que significa que o usuário dessa ferramenta teria certa aversão ao risco. O CVaR, também usado no trabalho, foi considerado como a média dos $5 \%$ piores resultados de contratação. Para simular o efeito do repasse à tarifa do consumidor final, utiliza-se, neste trabalho, o PLD de repasse (PLDrep). Neste caso, o valor do PLDrep ao longo dos anos é de 144,79 R\$/MWh. 


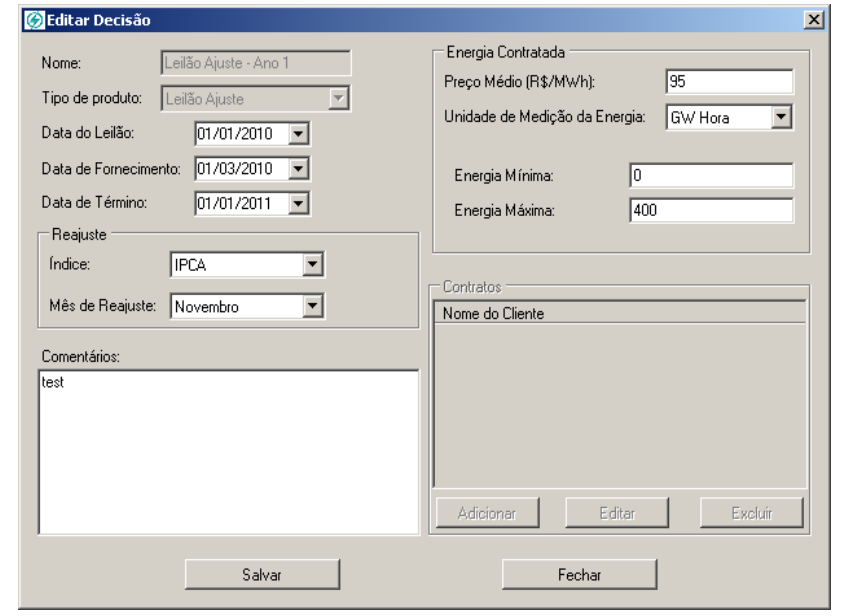

Figura 6: Interface gráfica com os parâmetros do leilão de ajuste no primeiro ano de decisão.

\subsection{Resultado da otimização sem a ferra- menta de otimização.}

Nesta subseção são apresentados os testes realizados simulando que a distribuidora opta por contratar quotas da energia disponível para contratação nos leilões A-1 e Ajustes, sem o uso do modelo proposto. Essas quotas variam de 0 (zero) até $100 \%$ da energia disponível. Na tabela 1 estão apresentados os resultados da contratação da distribuidora considerando as várias possibilidades de contratação.

Os resultados apresentados na tabela 1 indicam que a distribuidora obtém a melhor medida de custo (valor mais negativo) ao contratar $500 \mathrm{GWh}$ (50\% da quantidade disponível) dos leilões A-1 para cada ano e 400 GWh (100\% da quantidade disponível) dos leilões de Ajuste para cada ano de contratação. Este resultado também leva em conta o mecanismo financeiro para ajuste de sobras e deficts (MCSD Ex-post). O MCSD Ex-post é realizado no final do ano vigente, correspondendo até $20 \mathrm{GWh}$ da energia, em falta ou excesso, com preço para contratação de $80 \mathrm{R} \$ / \mathrm{MWh}$.

O resultado indica uma medida de custo de $-576,79$ milhões de reais. Os leilões de ajuste têm grande impacto relativo em períodos curtos de análises. São leilões com prazos de vigência menor (entre 10 e 24 meses). Assim, pode-se justificar uma contratação proporcionalmente alta em relação aos leilões A-1 por tratar-se de uma energia que pode ser mais facilmente ajustada ao longo do período de análise. Além disso, de acordo com os resultados, observa-se que a distribuidora estava (antes da decisão de contratação) relativamente sobrecontratada, pois não houve a necessidade de contratar toda energia disponível nos leilões A-1.

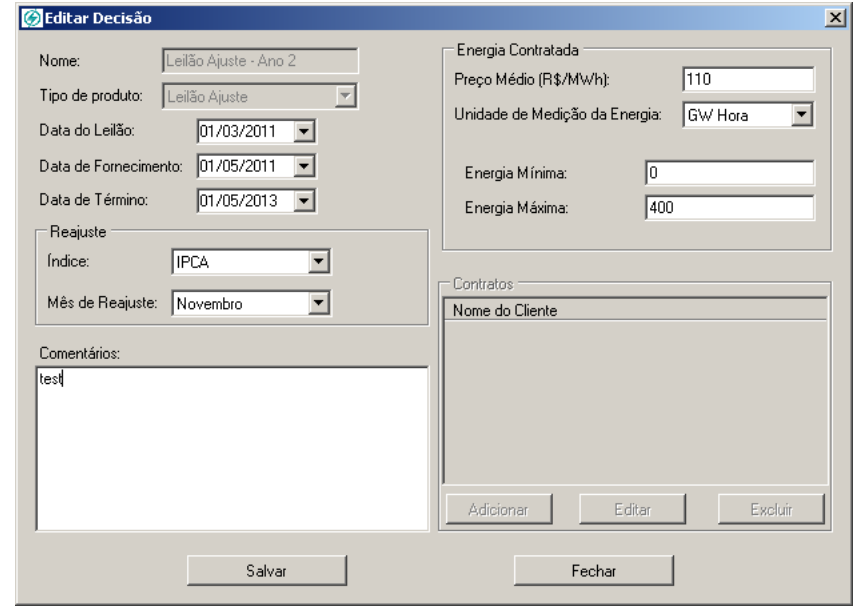

Figura 7: Interface gráfica com os parâmetros usados no leilão de ajuste para o segundo ano de decisão.

\subsection{Resultado da otimização com a ferra- menta de otimização.}

Nesta seção são apresentadas as análises realizadas considerando o uso do modelo de contratação proposto e as mesmas condições de contratação apresentadas na seção anterior, ou seja, até $1000 \mathrm{GWh}$ para A-1, no primeiro e segundo ano, e $400 \mathrm{GWh}$ para ajuste, para o primeiro e segundo ano. Também é avaliada a possibilidade da utilização dos mecanismos de contratação e descontratação de energia (MCSD4\%) e o mecanismo financeiro para ajuste de sobras e deficts (MCSD Ex-post). Para os casos de estudo com o MCSD4\%, a distribuidora poderá contratar (ou descontratar) até $4 \%$, dos contratos vigentes (este percentual também é otimizado). Já o MCSD Ex-post permite o ajuste financeiro no final do ano vigente de até $20 \mathrm{GWh}$ da energia, em falta ou excesso. $\mathrm{O}$ preço usado para contratação no MCSD Ex-post é de 80 $\mathrm{R} \$ / \mathrm{MWh}$.

Na tabela 2 são apresentadas quatro possibilidades de simulações com o modelo de contratação proposto para os leilões A-1 e Ajuste: na primeira, o cálculo da energia a ser contratada nos leilões A-1 e Ajuste é feito sem considerar o MCSD Ex-post e o MCSD4\%; na segunda simulação considera-se apenas a utilização do MCSD Ex-post; na terceira simulação apenas o MCSD4\% é considerado; finalmente, na quarta simulação MCSD Ex-post e MCSD4\% são considerados.

Na primeira simulação, apresentada na tabela 2, observa-se o quanto eficiente pode ser o sistema de otimização proposto. Para a contratação apresentada tem-se uma medida de custo de $-589,70$ milhões de reais, sendo maior que qualquer resultado obtido na tabela 1. Esse resultado foi conseguido 


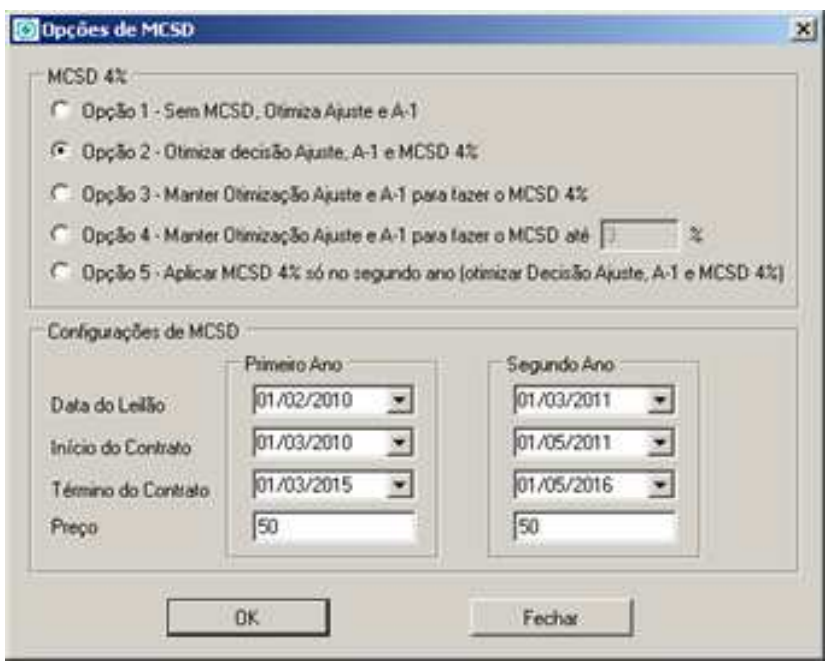

Figura 8: Interface gráfica usada para definir os parâmetros usados no MCSD 4\% e a configuração de otimização desejada.

a partir de uma expressiva contratação dos leilões de ajuste (97,46\% da energia disponível para o primeiro ano e 90,99\% para o segundo ando), e uma contratação relativamente mais baixa para os leilões A-1 (6,68\% e $0,43 \%$ para o primeiro e segundo ano, respectivamente). Esse resultado pode ser explicado pelo impacto relativamente maior do leilão de ajuste em relação ao leilão A-1 em um horizonte de análise relativamente curto. Além disso, novamente, tem-se um indicativo de que a distribuidora se encontra relativamente sobrecontratada, porque não contratou $100 \%$ da energia disponível.

O efeito positivo da contratação através do modelo proposto é potencializado quando se usa o MCSD Ex-post para eventuais ajustes financeiros. Com o uso desse mecanismo podese alcançar uma medida de custo de $-592,26$ milhões de reais, conforme é ilustrado na simulação 2 da tabela 2 .

$\mathrm{Na}$ segunda parte da tabela (simulações 3 e 4) são apresentados os resultados obtidos com o MCSD4\%. Os resultados mostram que o uso deste mecanismo produziu um valor de -621,94 milhões de reais, bastante superior ao resultado obtido sem o MCSD4\%. Esse resultado foi obtido com uma contratação de 2937,03 GWh, ou seja, quase três quartos da energia disponível para esta modalidade $(2,97 \%$ dos $4 \%$ de energia disponível) no primeiro ano e uma descontratação de $2540,81 \mathrm{GWh}(2,57 \%$ dos $4 \%$ da energia disponível para esta modalidade) dos contratos de energia existente para o segundo ano. O resultado líquido desta contratação $(2937,03$ $-2540,81=396,22 \mathrm{GWh}$ ), apesar de relativamente pequeno, produz um ganho muito superior a situação da distribuidora nas simulações 1 e 2 . Este fato se deve não somente devido
Tabela 1: Resultado por quotas de contratação da distribuidora e custo associado a contratação.

\begin{tabular}{|c|c|c|c|}
\hline $\begin{array}{l}\text { Cenários de } \\
\text { contratação }\end{array}$ & $\begin{array}{l}\text { Percentual } \\
\text { de contra- } \\
\text { tação A-1 } \\
\left(1^{\circ} \text { e } 2^{0} \text { ano }\right) \\
\text { (Total: } 1000 \\
\text { GWh) }\end{array}$ & $\begin{array}{l}\text { Percentual } \\
\text { de contrata- } \\
\text { ção Ajuste } \\
\left(1^{\circ} \text { e } 2^{\circ} \text { ano }\right) \\
\text { (Total: } 400 \\
\text { GWh) }\end{array}$ & $\begin{array}{l}\text { Custo as- } \\
\text { sociado } \\
\text { à distri- } \\
\text { buidora } \\
\text { (milhões de } \\
\text { R\$) }\end{array}$ \\
\hline 1 & $0 \%$ & $0 \%$ & -553.55 \\
\hline 2 & \multirow{3}{*}{$50 \%$} & $50 \%$ & -565.82 \\
\hline 3 & & $70 \%$ & $\begin{array}{l}-570.48 \\
\end{array}$ \\
\hline 4 & & $100 \%$ & -576.79 \\
\hline 5 & \multirow{3}{*}{$60 \%$} & $50 \%$ & -563.78 \\
\hline 6 & & $70 \%$ & -571.71 \\
\hline 7 & & $100 \%$ & -574.19 \\
\hline 8 & \multirow{3}{*}{$70 \%$} & $50 \%$ & -560.22 \\
\hline 9 & & $70 \%$ & -567.81 \\
\hline 10 & & $100 \%$ & -572.15 \\
\hline 11 & \multirow{3}{*}{$80 \%$} & $50 \%$ & -559.12 \\
\hline 12 & & $70 \%$ & -562.80 \\
\hline 13 & & $100 \%$ & -568.29 \\
\hline 14 & \multirow{3}{*}{$90 \%$} & $50 \%$ & -554.25 \\
\hline 15 & & $70 \%$ & -560.17 \\
\hline 16 & & $100 \%$ & -565.31 \\
\hline 17 & \multirow{3}{*}{$100 \%$} & $50 \%$ & -551.60 \\
\hline 18 & & $70 \%$ & -558.40 \\
\hline 19 & & $100 \%$ & -564.34 \\
\hline
\end{tabular}

à quantidade de energia líquida efetivamente contratada, mas também devido a sazonalização dos contratos obtidos com este mecanismo. A partir do resultado, nota-se o quanto o mecanismo pode ser uma ferramenta importante de apoio à decisão para as distribuidoras.

Com esses resultados pode-se inferir com maior precisão sobre a situação da carga da distribuidora. Verifica-se que nos primeiros anos, a distribuidora encontra-se subcontratada, por isto uma contratação de energia relativamente alta nos leilões e no MCSD4\% do primeiro ano. Já nos últimos anos de análise, verifica-se que a distribuidora está sobrecontratada, dado que houve uma contratação relativamente baixa no leilão A-1 e uma forte descontratação no MCSD4\%.

Finalmente, o melhor resultado (-622,19 milhões de reais) foi obtido com o uso do MCSD4\% e o MCSD Ex-post.

Na figura 9 estão apresentados os resultado da contratação usando o modelo de otimização proposto na configuração 4 da tabela 2. Neste caso é apresentado como o uso do modelo proposto ajusta os novos contratos aos contratos existentes.

Para analisar a figura 9, é interessante dividi-la em três partes: de janeiro de 2010 até dezembro de 2011; de janeiro de 2011 
Tabela 2: Resultado da contratação com sistema de otimização.

\begin{tabular}{|c|c|c|c|c|c|c|c|}
\hline \multirow{3}{*}{ Simulação } & \multicolumn{6}{|c|}{ Decisões } & \multirow{3}{*}{$\begin{array}{c}\text { Custo } \\
\text { (Milhões de } \mathrm{R} \$ \text { ) }\end{array}$} \\
\hline & \multicolumn{3}{|c|}{ Ano 1} & \multicolumn{3}{|c|}{ Ano 2} & \\
\hline & $\begin{array}{l}\text { A-1 } \\
(\mathrm{GWh})\end{array}$ & $\begin{array}{l}\text { Ajuste } \\
(\mathrm{GWh})\end{array}$ & $\begin{array}{l}\text { MCSD } \\
4 \% \\
(\mathrm{GWh})\end{array}$ & $\begin{array}{l}\text { A-1 } \\
(\mathrm{GWh})\end{array}$ & $\begin{array}{l}\text { Ajuste } \\
\text { (GWh) }\end{array}$ & $\begin{array}{l}\text { MCSD } \\
4 \% \\
(\mathrm{GWh})\end{array}$ & \\
\hline $\begin{array}{l}1 \text { - (Sem o MCSD Ex-post } \\
\text { e sem o MCSD4\%) }\end{array}$ & $\begin{array}{l}66.82 \\
(6.68 \%)\end{array}$ & $\begin{array}{l}389.83 \\
(97.46 \%)\end{array}$ & 0 & $\begin{array}{l}4.32 \\
(0.43 \%)\end{array}$ & $\begin{array}{l}363.94 \\
(90.99 \%)\end{array}$ & 0 & -589.70 \\
\hline $\begin{array}{l}2 \text { - (Com MCSD Ex-post e } \\
\text { sem o MCSD4\%) }\end{array}$ & $\begin{array}{l}309.48 \\
(30.95 \%)\end{array}$ & $\begin{array}{l}392.34 \\
(98.09 \%)\end{array}$ & 0 & $\begin{array}{l}0.13 \\
(0.01 \%)\end{array}$ & $\begin{array}{l}262.56 \\
(65.64 \%)\end{array}$ & 0 & -592.26 \\
\hline $\begin{array}{l}3 \text { - (Sem MCSD Ex-post e } \\
\text { com MCSD 4\%) }\end{array}$ & $\begin{array}{l}681.18 \\
(68.12 \%)\end{array}$ & $\begin{array}{l}151.88 \\
(37.97 \%)\end{array}$ & $\begin{array}{l}2937.03 \\
(2.97 \%)\end{array}$ & $\begin{array}{l}84.93 \\
(8.49 \%)\end{array}$ & $\begin{array}{l}365.98 \\
(91.50 \%)\end{array}$ & $\begin{array}{l}-2540.81 \\
(-2.57 \%)\end{array}$ & -621.94 \\
\hline $\begin{array}{l}4 \text { - (Com MCSD Ex-post e } \\
\text { com MCSD 4\%) }\end{array}$ & $\begin{array}{l}883.00 \\
(88.30 \%)\end{array}$ & $\begin{array}{l}173.80 \\
(43.45 \%)\end{array}$ & $\begin{array}{l}2957.55 \\
(2.99 \%)\end{array}$ & $\begin{array}{l}66.81 \\
(6.68 \%)\end{array}$ & $\begin{array}{l}368.30 \\
(92.08 \%)\end{array}$ & $\begin{array}{l}-2736.26 \\
(-2.77 \%)\end{array}$ & -622.19 \\
\hline
\end{tabular}

até maio de 2013, e, finalmente, de junho de 2013 até o final do período de análise.

Nesta figura, na primeira parte há uma forte contratação, dado que a distribuidora possui uma quantidade de energia contratada relativamente menor que os cenários de carga apresentados (subcontratada). Na segunda parte, observa-se um período que não há a necessidade de contratação. Neste período, o modelo de otimização estabelece que a quantidade de energia existente seja suficiente para cobrir a carga. Finalmente, na terceira parte, observa-se uma forte descontratação, resultado da aplicação do MCSD4\%. Neste período há um indicativo de que a distribuidora encontra-se sobrecontratada com relação a maior parte dos cenários de carga apresentados e, por isto, há a necessidade de descontratação. As três partes analisadas podem ser observadas mais claramente com a figura 10 .

Na figura 11 são apresentados os diferentes custos para os cenário de carga e a frequência de ocorrência destes custos. Os resultados apresentados na figura 11 estão relacionados com a simulação 4, apresentada na tabela 2 . Como se observa, para todos os cenários de carga possíveis a distribuidora obteria lucro, usando o modelo de contratação proposto. Neste caso, o lucro da distribuidora, obtido pela equação (7), para os cinco anos de análise, poderia variar entre 606 milhões de reais e 679 milhões de reais.

\section{CONCLUSÃO}

Neste trabalho foi apresentado um modelo de otimização para contratação de energia elétrica no curto prazo baseado em algoritmo genético e otimização robusta. Foram comparados o modelo de otimização proposto para contratação de energia no curto prazo e algumas simulações propostas de forma intuitiva. Os resultados apresentados indicam que, mesmo considerando cenários incertos de carga, o uso do PLD_robusto no modelo proposto visa proteger a distribuidora das variações do $P L D$ e garantir a contratação entre $100 \%$ e 103\% da carga. Assim, o uso do modelo proposto pode produzir ganhos significativamente maiores em relação às prováveis contratações apresentadas. De acordo com os resultados apresentados, o uso do modelo proposto é altamente recomendado nas situações nas quais se pretende usar o MCSD4\%. Neste caso, os resultados mostram que a ferramenta pode prover um ganho significativamente superior em relação às prováveis contratação e em relação ao uso apenas dos leilões A-1 e Ajuste. Também se mostrou que a utilização dos MCSD4\% e MCSD Ex-post juntos, além dos leilões de ajuste e A-1, produziram os melhores resultados para o estudo de caso apresentado. Entretanto, vale destacar que o uso do MCSD4\% pode prover ganhos significativamente maiores para as distribuidoras, no caso de poder contar com a disponibilidade da oferta de energia. Assim, nos próximos passos do trabalho serão analisados detalhadamente os efeitos do MCSD4\% na contratação de energia por parte das distribuidoras.

\section{AGRADECIMENTOS}

Os autores agradecem a Light Serviços de Eletricidade S.A, pela parceria no projeto SIGRICE no P\&D ANEEL 37/07.

\section{REFERÊNCIA BIBLIOGRÁFICA}

ANEEL - Resolução Normativa ANEEL No 5163, de 30 de julho de 2004.

ARTZNER, P; DELBAEN, F; EBER, J-M AND HEATH, D. "Coherent measures of risk". Mathematical Finance, (3), 203-228, 1999. 


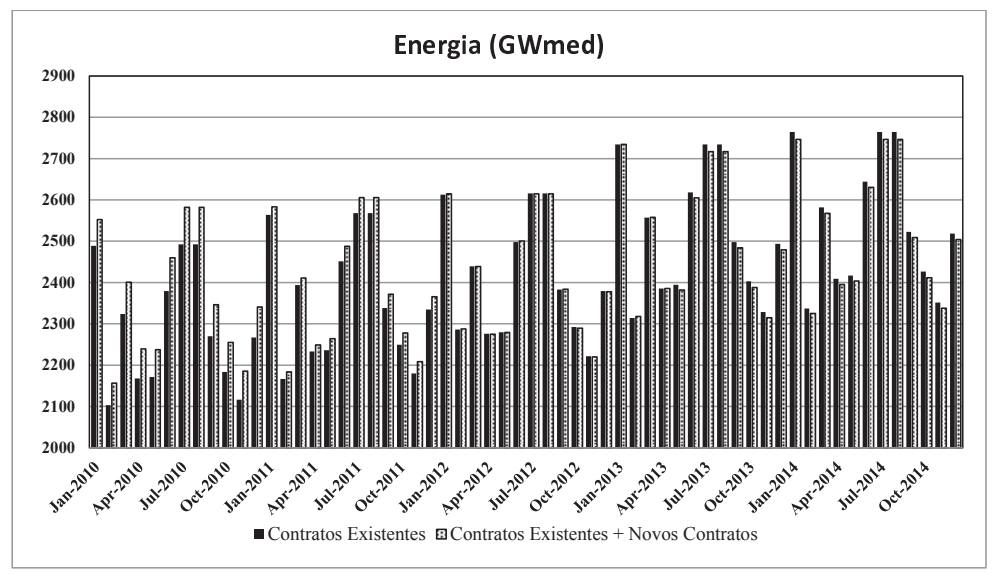

Figura 9: Configuração temporal dos contratos da distribuidora antes e após o processo de otimização.

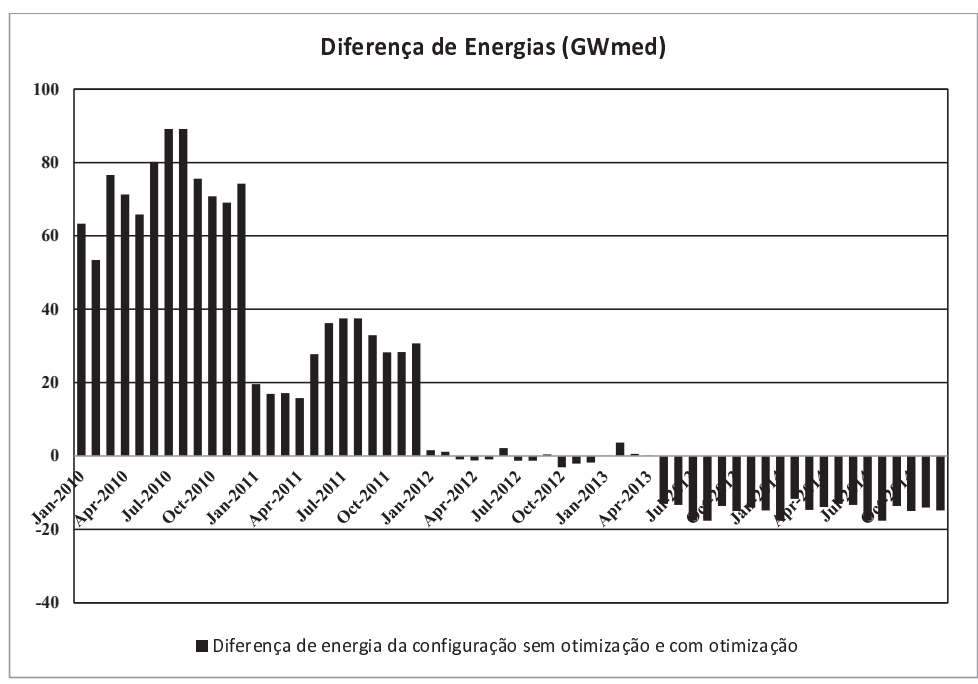

Figura 10: Diferença (GWmed) entre contratos existentes e o resultado da otimização dos contratos existentes.

AZEVEDO, E. M. "Modelo Computacional de Teoria dos Jogos aplicado aos Leilões Brasileiros de Energia Elétrica". Tese de doutorado. Faculdade de Engenharia Mecânica, Universidade Estadual de Campinas, 2004.

BARROSO, L. A; STREET, A; FLACH, B and VEIGA, M. P. ."Offering Strategies and Simulation of Multi-Item Iterative Auctions of Energy Contracts". IEEE Transactions on power systems. Aceito para publicação em 2011.

BENATI, S., RIZZI, R., A mixed integer linear programming formulation of the optimal mean/Value-at-Risk portfolio problem. European Journal of Operational Research, vol. 176, pp. 423-434, 2007.
BIRGE, J.R. "Quasi-Monte Carlo Methods Approaches to Option Pricing". Technical report 94-19., Department of Industrial and Operations Engineering, The University of Michigan, 15p.

BOYLE, P., BROADIE, M., GLASSERMAN, P (1997). Monte Carlo Methods for Security Pricing, Journal of Economic Dynamics and Control, Vol.21, 1997, pp.1267-1321.

CASTRO, M. A. L. "Análise dos Riscos de uma Distribuidora Associados à Compra e Venda de Energia no Novo Modelo do Setor Elétrico". Dissertação de Mestrado em Engenharia Elétrica. Universidade de Brasília, 2004.

CASTRO, R. "Análise de Decisões sob Incerteza para Investimento e Comercialização de Energia Elétrica no 


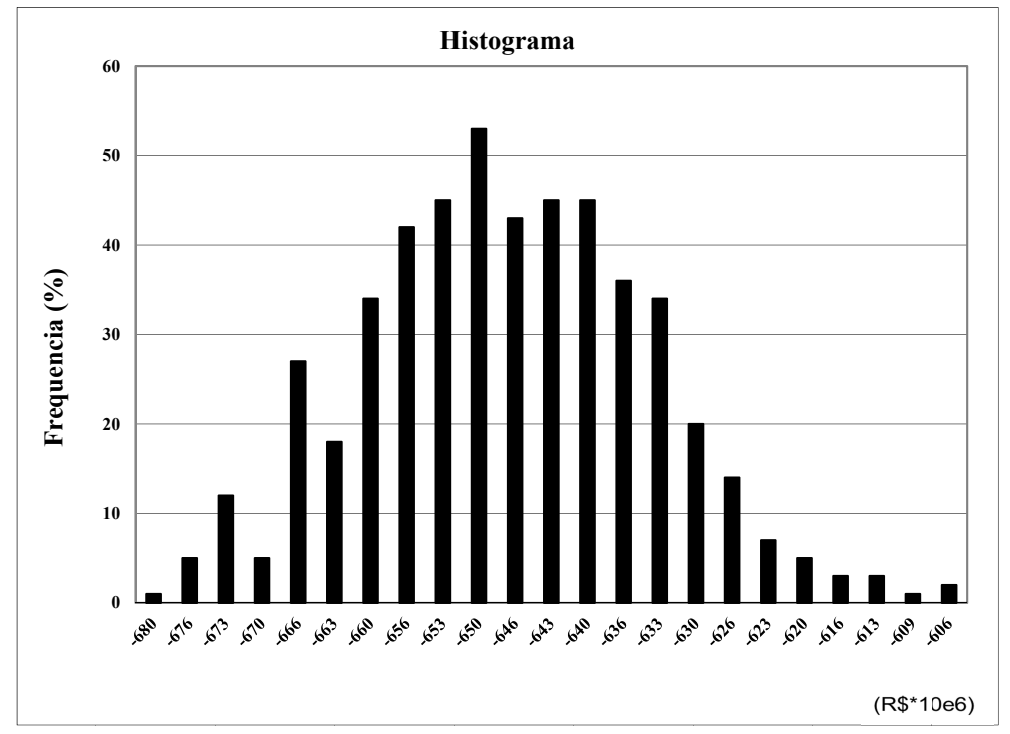

Figura 11: Histograma de custos (em milhões de reais) da distribuidora.

Brasil". Tese de Doutorado em Engenharia Elétrica, Universidade Estadual de Campinas, 2004.

CCEE - Câmara de comercialização de energia elétrica. Acesso online: www.ccce.com.br, em janeiro de 2010.

DECRETO No 5.163. "Regulamenta a comercialização de energia elétrica, o processo de outorga de concessões e de autorizações de geração de energia elétrica”. Brasília, julho de 2004.

GOLDBERG, DAVID E. 'Genetic Algorithms in Search, Optimization, and machine Learning”. Addison-Wesley Publishing Company, Inc, 1989.

GUDER, R. "Otimização de portifólios de contratos de energia elétrica utilizando algoritmos genéticos multiobjetivo". Dissertação de Mestrado. Universidade Federal de Santa Catarina, 2009.

GUIMARÃES, A. "Estratégias de Contratação das Distribuidoras sob Incerteza de Demanda em Leilões de Energia”. Dissertação de Mestrado, Pontifícia Universidade Católica do Rio de Janeiro, 2005.

HUANG, X. Portfolio selection with a new definition of risk. European Journal of Operational Research, vol. 186, pp.351-357, 2008.

ISHIHARA, C. A. "Utilização de derivativos financeiros na mitigação dos riscos de comercialização de energia elétrica no Brasil”. Dissertação de Mestrado, Universidade de Brasília, 2002.
JORION, P. "Value at Risk. A nova fonte de referência para o controle de risco de Mercado". São Paulo, Bolsa de Mercadorias \& Futuros, 1998.

LU, G.; WEN, F.; CHUNG, C.Y.;WONG, K.P. Conditional Value-at-Risk Based Mid-Term Generation Operation Planning in Electricity Market Environment. IEEE Congress on Evolutionary Computation, 2007.

LAZO, J.; FIGUEIREDO, K; LIMA, D.A; SILVA, C e Aor, L. "Sistema inteligente de contratação de energia elétrica no curto prazo". CBA, Bonito-MS, 2010.

MICHALEWICZ, Z. "Genetic Algorithms + Data Structures = Evolution programs". Springe-Verlag, USA, 1996.

MME - Ministério das Minas e Energia. Modelo institucional do setor elétrico, Brasília, dezembro de 2003.

OLIVEIRA, E. M. "Estratégia de comercialização de energia elétrica através da otimização de portifólios de contratos utilizando projetos de experimentos de misturas". Tese de doutorado. Universidade Federal de Itajubá, 2009.

ROCKAFELLAR, R. T. AND URYASEV, S. P. “ Optimization of Conditional value at risk". The journal of risk, vol. 2, No 3, pp. 21-41, 2000.

RODRIGUES, F. F. C; FALCÃO, D. M. AND BORGES, C. L. T. "Programação da contratação de energia considerando geração distribuída e incertezas na previsão de demanda". Revista Controle \& Automação/Vol.18 no.3/Julho, Agosto e Setembro 2007. 
SILVA, N. L. A. "Derivativos: Definições, Emprego e Risco. São Paulo, Atlas, 1998.

SUSTERAS,G.L. "Aplicação de Algoritmos Genéticos para previsão do comportamento das distribuidoras como apoio à estratégia de comercialização de energia de Agentes Geradores". Dissertação de Mestrado, Escola Politécnica, Universidade de São Paulo, 2006.

STREET, A; BARROSO, L. A; FLACH, B and VEIGA, M. P. "Risk Constrained Portfolio Selection of Renewable Sources in Hydrothermal Electricity Markets". IEEE Transactions on power systems, vol. 24, no. 3, August 2009.

SOBOL, I.M. "On the Distribution of Points in Cube and the Approximate Evaluation of Integrals". U.S.S.R Computational Mathematics and Mathematical Physics, Vol. 7, no. 4, pp. 86-112, 1967.

WANG, X. "Variance Reduction Techniques and QuasiMonte Carlo Methods", Journal of Computational and Applied Mathematics, $\mathrm{N}^{\mathrm{o}}$ 132, pp.309-318, 2001.

TORRES, R. "Avaliação de portfolios de contratos de compra e venda de energia elétrica: Uma abordagem pela teoria de opções". Dissertação de Mestrado. Pontifícia Universidade Católica, 2006. 